INVESTIGACIÓN/RESEARCH

\title{
EMPODERAMIENTO DE LAS REDES SOCIALES EN LAS CRISIS INSTITUCIONALES
}

Mercedes Cancelo Sanmartín¹. Universidad de Málaga. España. mcancelo@gmail.com

Gilda Gadea Aldave. Universidad Autónoma de Tamaulipas. México.

ggadeaa@gmail.com

\section{RESUMEN}

En el presente artículo se pretende ahondar en el uso de las redes sociales como vehículo comunicativo veraz ante el vacío comunicativo institucional, concretamente en situaciones de riesgo para la sociedad civil. Desde la concepción de la existencia institucional se plantea como inherente el accionar estatal de protección de los ciudadanos y como consecuencia la difusión de aquellos mensajes que puedan contribuir a la seguridad de la ciudadanía. Cuando la emisión de mensajes en estas circunstancias no es proactivo o incluso es inexistente, los ciudadanos buscan organizarse como fuentes de información reactivas a la situación de riesgo en la que perciben vivir. Para la constatación de la existencia de estas fuentes de información paralelas y su uso ciudadano, se han estudiado tres regiones de México que actualmente atraviesan situaciones de inseguridad ciudadana, concretamente los estados de Nuevo León, Tamaulipas y Veracruz. La metodología aplicada ha sido la encuestación de un número significativo de ciudadanos divididos equitativamente entre las capitales de los tres estados citados anteriormente. Los resultados más relevantes apuntan hacia la falta de legitimación de las fuentes tradicionales de información en pos de las alternativas promovidas por los propios ciudadanos.

PALABRAS CLAVE: Comunicación - Institucional - Redes - Inseguridad - Medios Ciudadanía

\footnotetext{
1 Autor correspondiente:

Mercedes Cancelo Sanmartín: Profesora del Departamento de Comunicación Audiovisual y Publicidad en la Universidad de Málaga. España.

Correo: mcancelo@gmail.com
} 


\title{
EMPOWERMENT OF SOCIAL NETWORKS IN INSTITUTIONAL CRISIS
}

\begin{abstract}
This paper aims to delve into the use of social networks as communication vehicle truthful communication to the institutional vacuum, particularly in situations of risk to civil society. From the conception of institutional organizational existence arises as state action inherent protection of citizens and consequently spreading messages that could contribute to the public safety. When the broadcasting of messages in these circumstances is not proactive or even there, citizens seeking information sources organized as reactive to the risk they perceive life. For the establishment of the existence of these sources and their use parallel citizen, studied three areas of Mexico currently grappling situations of insecurity, particularly the states of Nuevo Leon, Tamaulipas and Veracruz. The methodology has been applied in alms of a significant number of citizens divided equally between the capitals of the three states mentioned above. The most relevant results point to the lack of legitimacy of traditional sources of information towards the alternatives promoted by the citizens themselves.
\end{abstract}

KEYWORDS: Communication - Institutions - Networking - Insecurity - Media Citizenship

\section{INTRODUCCIÓN}

Las instituciones estatales en un ámbito democrático poseen una actividad bicéfala, por una parte deben estructurar la sociedad asegurando el correcto cumplimiento de los preceptos legales adquiridos socialmente y por otra parte son los responsables de garantizar la paz social en su amplio significado. En determinados casos la paz social no solo responde a acciones puramente instrumentales en cuanto a desarrollo institucional, sino que asimismo se enfatiza la generación de mensajes que apoyen el propio desarrollo institucional y social. Por desarrollo institucional se entiende el desempeño de la labor propia del ente gubernamental con el fin de conseguir los objetivos marcados. Respecto al desarrollo social comprendemos la labor inherente a la propia institución que la consigna como un servicio público que otorgue respuestas a las demandas ciudadanas. Por lo tanto se entiende socialmente la labor de las instituciones públicas como fuentes que aseguran el mantenimiento de la estructura social y a su vez establecen espacios de información para garantizar el desarrollo democrático de su labor (Sauvy, 1970:122).

Las instituciones cumplen la función pública encomendada por el gobierno de un país, lo cual significa que estos entes son las extensiones gubernamentales que cumplen una 
función instrumental y además conforman la imagen pública ${ }^{2}$ del gobierno activo. Consecuencia de ello es que los ciudadanos perciben a las instituciones públicas como representación del gobierno y arma ejecutora de las políticas gubernamentales aprobadas por el mismo.

Debido a esta traslación del valor de la imagen del poder en vigor a las instituciones, resulta inevitable que la legitimación que se realice a las instituciones se traslade a los gobiernos. "Legitimidad dada por el desarrollo de la institución de acuerdo con unas creencias, normas y valores" como dice Sotelo Enríquez (2001:27). Se entiende que la institución se conforma en una sociedad con arreglo a una cultura y a un desarrollo social concreto. La organización se concede la condición de legítima al integrarse y pervivir en el universo social. Una institución no sólo debe administrar eficazmente sus recursos sino también integrarse en el universo cultural de la sociedad. También se destaca en la definición el hecho de que la institución dependerá de los principios configuradores. Dichos principios están compuestos por principios legales, principios morales y principios formativos. Estos últimos son los que diferencian a las organizaciones. Cada una emite y posee una información distinta que aportar a la sociedad y al resto de organizaciones.

Hablar de aceptación significa hablar de legitimación. Weber (1984:701) se refiere a la base de la legitimidad como "la vigencia del poder, es decir, la pretensión de que el gobernante sea obedecido por sus funcionarios y todos ellos por los gobernados"

Este no es un proceso voluntario, sino inducido a través de un conjunto de ideas de un colectivo; el vínculo entre estas ideas con los actores gubernamentales, y la aceptación o transformación a nuevas temáticas que pasan a formar parte de la opinión pública, fiscalizadas a su vez por el liderazgo de determinados actores, que participarán activamente en el proceso. Esto es posible a través del proceso de comunicación desarrollado por las instituciones, instrumentado por los medios de comunicación y recibido por los ciudadanos.

\subsection{Fuentes de información y comunicación institucional.}

El desarrollo de la institución debe estar vinculado intrínsecamente al de su comunicación. Tal y como se mencionaba con anterioridad la comunicación está ligada a la propia función de la institución. Incluso el desarrollo comunicativo del ente repercute directamente en dos aspectos fundamentales de su existencia: la reputación y la autoridad. Ambos son vinculantes y establecen el principio de legitimación institucional. De la Porte (2012: 281-282) alude a la reputación institucional señalando que es un proceso que conlleva un tiempo de desempeño institucional y posterior evaluación ciudadana. Igualmente señala que la autoridad es un significante necesario en el desarrollo institucional ya que a través de su ostentación los entes

\footnotetext{
2 Defiendo imagen pública como la percepción de los públicos de la institución. La percepción se compone de los siguientes factores: actos de la institución, relación con los ciudadanos y opiniones o noticias vertidas por los medios de comunicación.
} 
gubernamentales pueden desarrollar su labor instrumental es decir, cumplir el objetivo que justifica su creación.

En situaciones de crisis social, como por ejemplo las amenazas que irrumpen suspendiendo la seguridad entre la población, la comunicación institucional se convierte en un necesidad vital desde el punto de vista ciudadano. En esas circunstancias, como las que se aluden en la presente investigación, el acceso a la comunicación que ofrecen las instituciones en el cual impera la "interacción", no asegura el acceso social a la información (Bolz, 2006:8). Precisamente porque la información dispensada no responde a las demandas sociales puntuales en pos de la situación del entorno. Durandin (1996:193) indica que en situaciones de crisis "las personas inquietas, buscan informaciones que les ayuden a tomar decisiones". Las fuentes legitimadas de información en una sociedad democrática son las instituciones públicas. Estas a través de su comunicación dan a conocer el estado de la cuestión y la relación existente con el riesgo real a la población civil

Normalmente ante vacíos comunicativos institucionales, los medios de comunicación preponderan su papel de vigilancia (siguiendo la huella de los actores políticos) y de correlación (organizando respuestas hacia ellos). En esa instancia los ciudadanos encuentran en los medios la fuente de información que previamente buscaron en los entes estatales (Price, 2001: 108). Pero el problema en este eslabón aparece cuando la percepción de la comunicación desea sustituir a la percepción del mundo es decir, los medios tradicionales no cumplen el desarrollo de sus funciones activamente y solo reflejan una imagen previamente trasladada por las instituciones públicas (Bolz, 2006: 12).

En este sentido es importante recordar que Noelle-Neumman (2005:67) destaca la construcción de la opinión pública utilizando a los medios de comunicación como "creadores" de crisis y novedades, en base a las diversas temáticas abordadas por los mismos, estos competirán unos con otros por la atención de los públicos y para posteriormente formar parte de la opinión pública. Conjuntamente con esta visión novedosa de la opinión pública en la cual se enfoca la atención en la creación de situaciones que producen alteraciones sociales, vinculamos a Lippman (1922: 29) que señala que "los ciudadanos forman sus ideas a partir de informaciones gravemente incompletas manteniendo poco o nulo contacto con los hechos reales". Ambos autores enfocan la construcción de la realidad a través de medios de comunicación que son abastecidos en parte de información a través de las instituciones públicas, dejando por ende en estas últimas la responsabilidad de entregar un producto veraz y acorde con el marco real de la sociedad en cuestión.

\subsection{Las redes sociales como fuente de información}

Internet significa en muchos casos el acceso a información no suministrada por las fuentes activas y legítimas de información. Se ha convertido por lo tanto en un emisor de mensajes paralelos que introducen información nueva o reactiva a las demandas de sus usuarios. En el caso de México en el año 2012 según el Instituto Nacional de Estadística y Geografía mexicano un total de 45 millones de ciudadanos han accedido 
de forma regular a la información en la red, lo que significa un incremento de $27 \%$ respecto al año anterior.

Podemos observar cierta relación entre la falta de información institucional en situaciones de inseguridad y el crecimiento del auge del uso de internet en el país citado. Precisamente en esta investigación se alude al establecimiento de un vínculo entre el uso de internet y el vacío de comunicación institucional. Debido principalmente al planteamiento de qué hacen los ciudadanos cuando no reciben información respecto a situaciones de crisis en el entorno social, como es el caso de la inseguridad ciudadana. La acción ciudadana se centra entonces en la búsqueda y consumo de fuentes informales de información. Es decir, fuentes que trasmiten datos corroborados y verídicos de los sucesos que les interesan a los diversos públicos que conforman la sociedad, que son comprobables e incluso exhiben fotografías de los hechos y que a través de la aceptación de los usuarios se han ganado la legitimación como fuentes confiables de información (Rivas, 1995:78).

En el caso de México las fuentes informales están siendo encarnadas por las redes sociales, defiendo a estas como "conjunto de personas que interactúan entre sí, de modo que cada miembro recibe la influencia de cada una de las otras personas y a su vez, ejerce influencia en ellas" (Fernández, 2001:15). A diferencia de las redes sociales físicas o tradicionales, las redes sociales en internet presentan un acceso directo entre usuarios. Destacando especialmente las acciones de comunicación directa, cooperación y creación de comunidad.

En vista de lo señalado cobra importancia conocer la legitimación ciudadana a estas nuevas fuentes de información. Las redes sociales, erigidas en situaciones de inseguridad ciudadana ante el vacío informativo institucional y mediático.

\section{METODOLOGÍA}

La investigación realizada se enfocó en conocer el grado de necesidad de información que demandan los ciudadanos en función de la percepción de riesgo social. Para ello se tomó como grupo muestra un conjunto de ciudadanos ubicados en las tres urbes que conforman las capitales de los estados de Nuevo León, Tamaulipas y Veracruz es decir, Monterrey, Ciudad Victoria y Xalapa. La elección territorial se basó en la revisión de fuentes mediáticas ${ }^{3}$ en las cuales se alude a que las citadas regiones están atravesando situaciones de alarma social por disturbios en materia de seguridad ciudadana. Se consideraron tres sectores de cada ciudad segmentándolas en función de sus pobladores bajo las características determinantes de su clase social y nivel sociocultural, con el fin de conocer si el nivel de riesgo y demanda de información se relacionaba con estos dos factores de definición social. El número toral de encuestas aplicadas fue de 3600 encuestas, señalando que se han realizado 1200 encuestas en cada capital de estado, a continuación se han baremado un número de 400 encuestas por grupo poblacional. Entre las 400 encuestas aplicadas por sector y nivel social, se

\footnotetext{
${ }^{3}$ Diario Universal (México), Univisión (USA), El País (España), Clarín (Argentina) entre otros.
} 
buscó la equidad de género procesando de tal modo 200 encuestas a hombres y un número igual a mujeres. El número total de preguntas fue de 10 con respuestas desglosadas en opciones. La franja de edad elegida fue entre 20 y 50 años, buscando con este parámetro establecer una horquilla de edad activa que consume altamente medios de comunicación y que posee un conocimiento proactivo de la noticiabilidad y de su micro entorno. Las encuestas fueron aplicadas entre los meses de octubre de 2011 y junio de 2012.

El primer objetivo de investigación ha sido conocer la percepción de inseguridad ciudadana en los Estados de México analizados, teniendo en cuenta el vacío de comunicación institucional al respecto. El segundo objetivo planteado fue detectar el grado de legitimación institucional en situaciones de riesgo percibido por la ciudadanía. El tercer objetivo es conocer si las redes sociales se han convertido en fuentes activas de información ante la percepción de inseguridad y el silencio comunicativo procedente de los entes de información tradicionales. Como último objetivo se planteó establecer si el creciente uso de internet se produce como fuente paliativa del vacío informativo en medios y fuentes tradicionales.

\section{ANÁLISIS Y DISCUSIÓN.}

El desarrollo del estudio se centró en conocer el grado de respuesta que detectan los ciudadanos a las demandas de información en situaciones de inseguridad y las posibles alternativas de fuentes de información que encuentran en su búsqueda reactiva. Para ello en primer lugar es necesario destacar que la mayoría de los ciudadanos encuestados detectaron una situación de riesgo y por lo tanto de alarma social en materia de seguridad ciudadana tal y como muestra el cuadro siguiente.

Gráfico 1. ¿Percibe situaciones de riesgo en la comunidad?

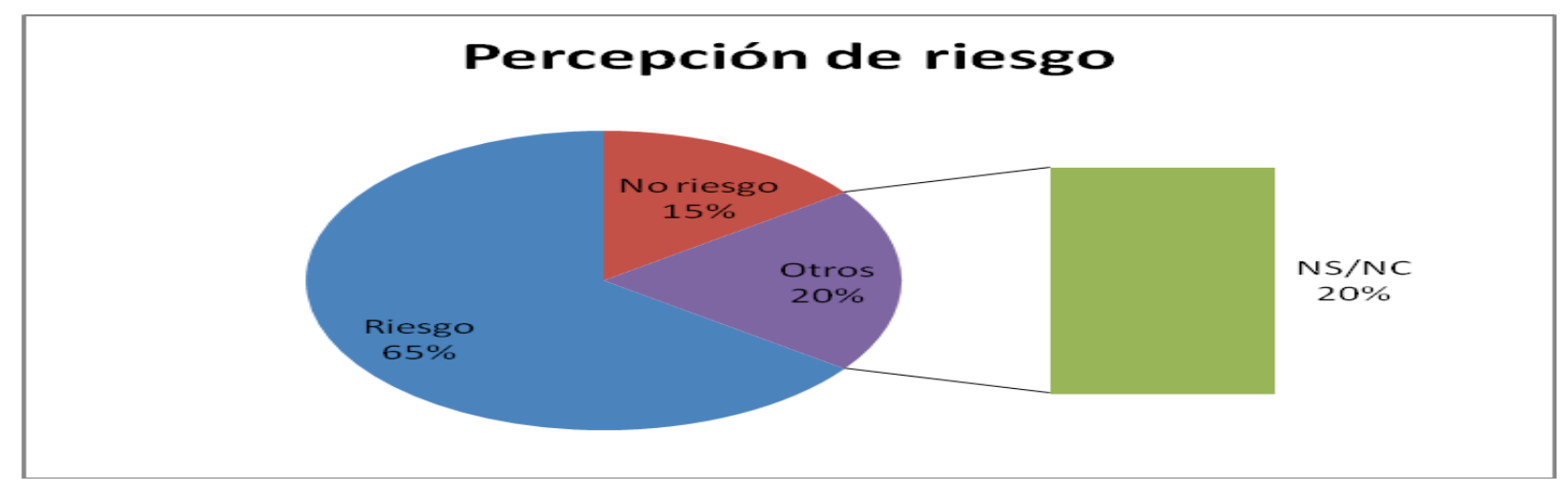

Fuente: Elaboración propia.

Respecto al riesgo detectado a nivel ciudadano se pregunta sobre la identificación que encuentran en la comunicación e información que dispensan los entes públicos y los medios de comunicación tradicionales. 


\section{Gráfico 2. ¿Encuentra referencias al riesgo percibido en los medios y en las instituciones públicas?}

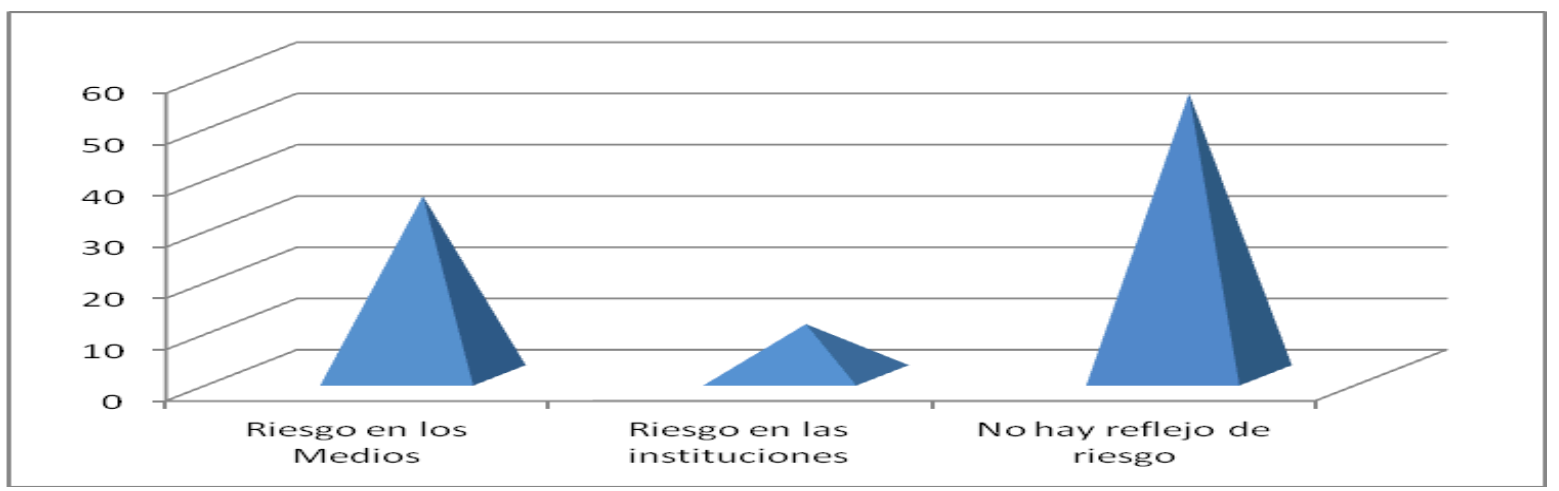

Fuente: Elaboración propia.

Asimismo se estableció la interrogante sobre cuales consideran los ciudadanos encuestados los vehículos de información fiables en situaciones de riesgo social. Buscando con esta pregunta establecer el nivel de legitimación que poseen los entes gubernamentales y los medios tradicionales en de comunicación.

Gráfico 3. Ante la detección de un riesgo, ¿cuál es para Usted un vehículo/fuente legítimo de emisión/transmisión de mensajes?

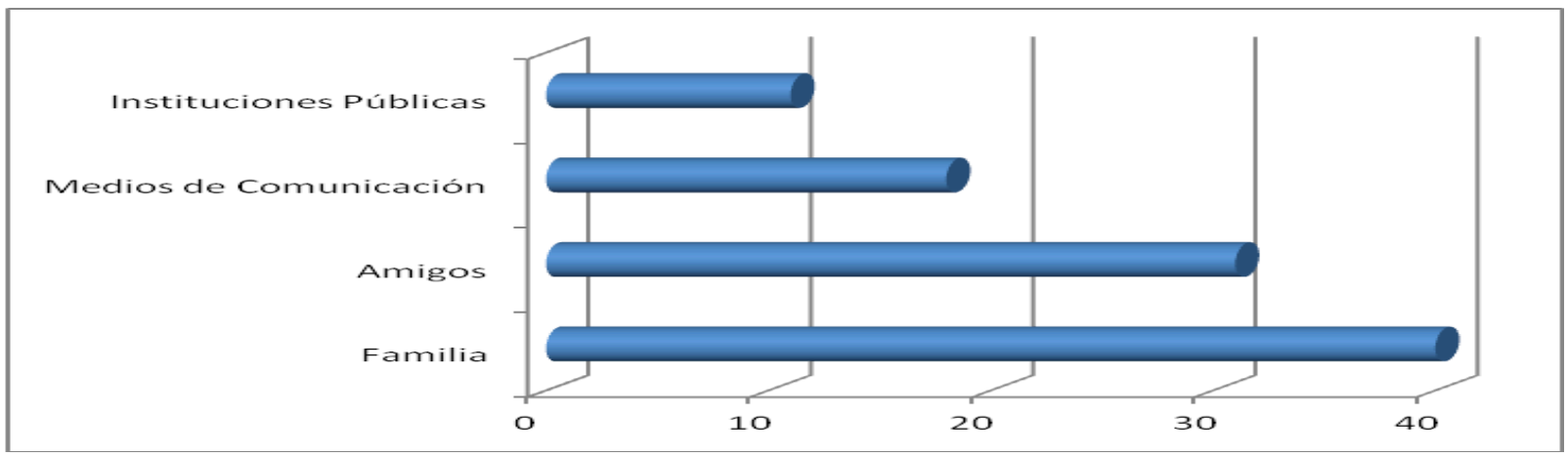

Fuente: Elaboración propia.

Respecto al consumo y credibilidad de los medios de comunicación se les planteó a los encuestados que evaluasen la credibilidad de los mismos como fuentes de información en sustitución de las gubernamentales.

Gráfico 4. ¿Qué medio considera que se ajusta más al reflejar la realidad? 


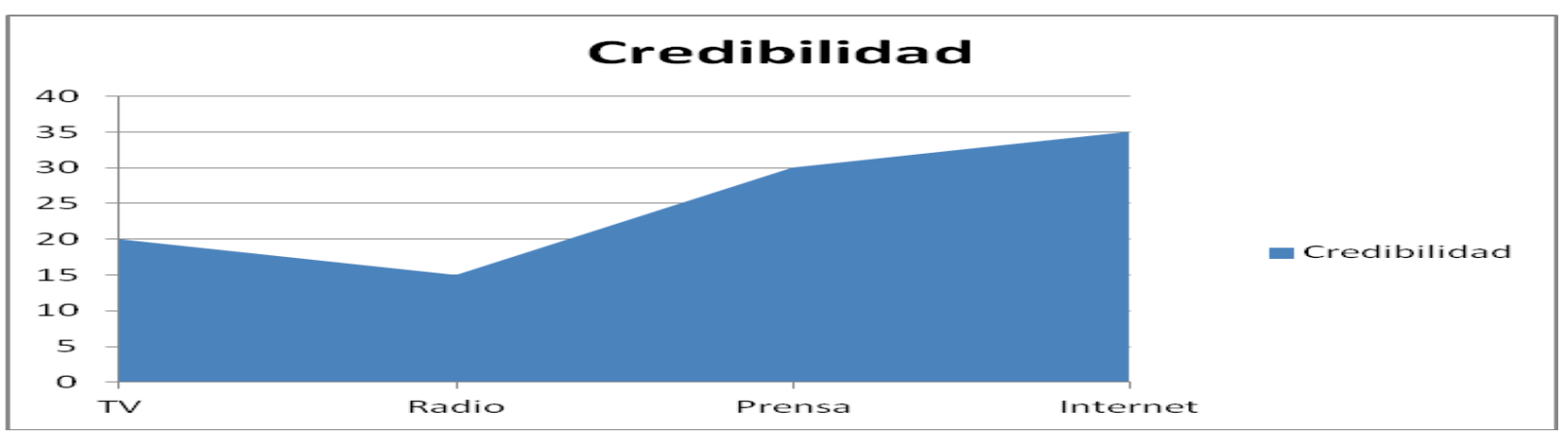

Fuente: Elaboración propia.

Resulta crucial conocer el uso de internet como alternativa comunicativa en la percepción de riesgo ciudadano, para ello se les preguntó a los encuestados si habían incrementado el uso de la red para obtener información en situaciones de inseguridad.

Gráfico 5. ¿Ha incrementado el uso de internet para buscar información sobre inseguridad?

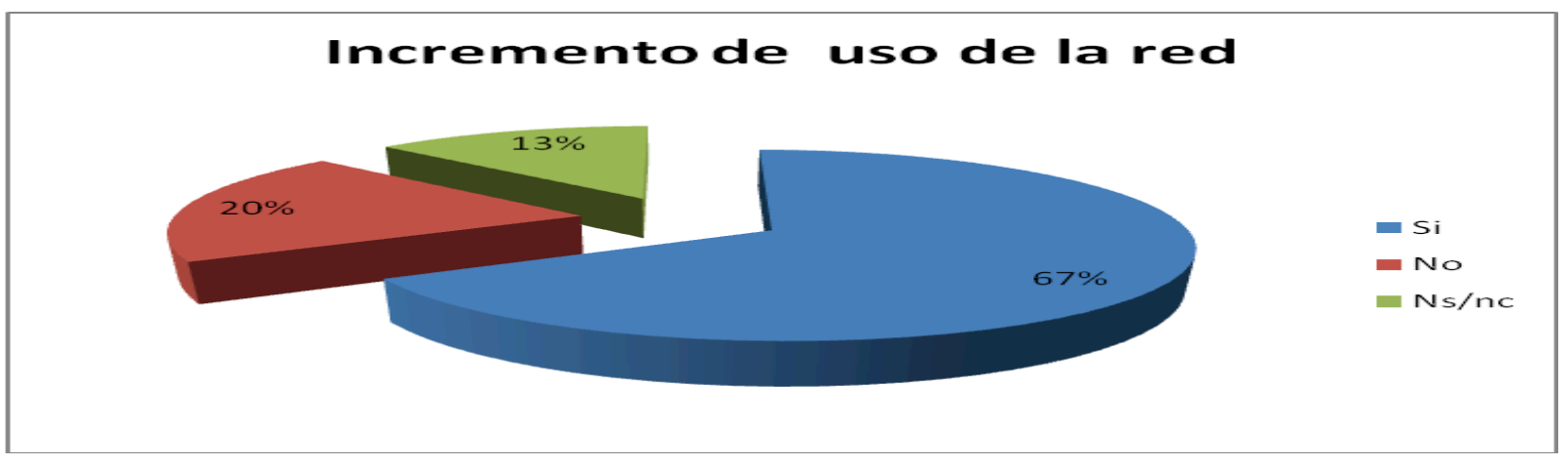

Fuente: Elaboración propia.

Por último se ha buscado conocer que elementos se consumen en internet, con el fin de establecer que fuentes dentro de la red gozan de legitimación en este tipo de información.

Gráfico 6. En su búsqueda de información en internet respecto a inseguridad, ¿cuál es la fuente más fiable y consultada por usted? 


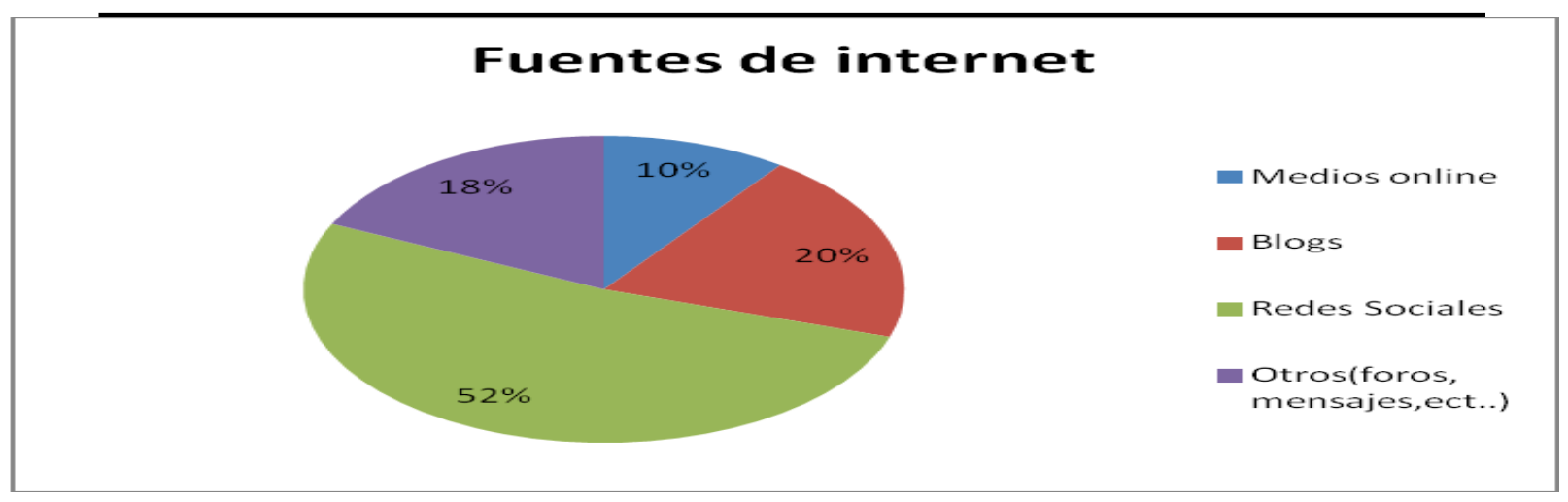

Fuente: Elaboración propia.

\section{CONCLUSIONES}

Mills (1956:304) aseveraba que "la realización de la opinión en acción está controlada por las autoridades, que organizan y controlan los canales de acción". Estableciendo por tanto el control de las fuentes de información legítimas en un estado democrático. Precisamente en democracia la comunicación es el garante de la transparencia organizacional, desprendiéndose de ello los entes institucionales deben responder con información a las necesidades de la ciudadanía.

En la investigación expuesta se presenta la necesidad de información ciudadana ante la percepción de hechos que amenazan la seguridad pública. Esta demanda proactiva obtiene el silencio informativo como repuesta institucional. Provocando por lo tanto que la atención informativa se centre en los medios tradicionales como transmisores de información. Lippman (1922:13) indicaba que "el ciudadano llega a sentirse como un espectador sordo de la última fila, que debiera mantener su atención fija en la trama general, pero además apenas puede conseguir mantenerse despierto". Según la aseveración de uno de los estudiosos de la formación de la opinión pública, los medios no cumplen su función de reflejar fielmente la realidad, entrando de este modo en un juego de construcción de la percepción en la cual las instituciones públicas brindan una visión intencionada.

Los ciudadanos encuestados encontraban situaciones que ellos consideraban de riesgo social e indicaban la ausencia de eco en los medios de comunicación ni indicación de actuación por parte de las instituciones públicas, generándose de este modo un vacío de acción pública.

La falta de constatación informativa en los medios de comunicación y la carencia de información por parte de los entes gubernamentales, han propiciado que los ciudadanos busquen otras fuentes paralelas de apoyo como la familia y los amigos. Generándose de este modo lo que se denomina como redes de apoyo social, a través de las cuales los individuos encuentran no sólo fuentes de contención a su miedo sino además un prisma más amplio de juicio ante la posible actuación individual. 
Internet, concretamente las redes sociales se han convertido en instrumento ciudadano para difundir y recibir información acerca de temas que consideran de interés social. Como es el caso de la inseguridad, las redes sociales están actuando como espacio de encuentro en el cual la ciudadanía interactúa convirtiéndose en emisor y receptor, generando por lo tanto un espacio de comunicación en paralelo con el tradicional (instituciones y medios de comunicación). En este sentido es importante señalar un riesgo existente en la búsqueda paralela de información por parte de la ciudadanía ante temas de su preocupación ya que no siempre puede considerarse de calidad. Como indicaba Mills (1956:304) "en una masa son muchas menos las personas que emiten una opinión que las que la reciben...las comunicaciones que prevalecen están tan organizadas que es difícil que el individuo responda ante el efecto....la realización de la opinión en acción está controlada por las autoridades que organizan y controlan los canales..."

Por otra parte existe una clara relación entre el incremento de uso de internet y la percepción de inseguridad ciudadana en México. Evidenciando la inactividad comunicativa de las fuentes históricamente legitimadas como de información: los entes gubernamentales y los medios de comunicación.

En conclusión podemos enunciar que las redes sociales están siendo legitimadas por los ciudadanos mexicanos como fuentes de información activa ante la percepción social de inseguridad. En este caso los agentes de información y los receptores de la misma son un solo ente (la sociedad civil) que busca soluciones proactivas al silencio institucional y mediático. Este hecho coloca en suspenso el papel del espacio público y la construcción de la opinión pública como habitáculo inmaterial de encuentro entre los distintos agentes sociales.

\section{REFERENCIAS}

Barroso, P. (1984). Códigos deontológicos de los medios de comunicación. Madrid: Ediciones Paulinas.

Bartoli, A. (1992). Comunicación y organización: la organización comunicante y la comunicación organizada. Barcelona: Paidós.

Beato Espejo, M. (2002). Cauces comunicativos de las administraciones públicas. Madrid: Tecnos.

Beck, A. \& Van Loon, J. (2000).The Risk Society and Beyond: Critical Issues for Social Theory. London: Sage.

Bolz, N. (2006). Comunicación mundial. Buenos Aires: Katz.

Blumer, H. (1966). The mass, the public, and the public opinion. New York: Free press.

Canel, M.J. (1999). Comunicación política. Técnicas y estrategias para la sociedad de la información. Madrid: Tecnos. 
Cathala, H.P. (1986). Le temps de la desinformation. Paris: Stock.

Crespi, I. (2000). El proceso de opinión pública. Madrid: Ariel.

Del Rio, P. (1996). Psicología de los medios de comunicación. Madrid: Síntesis.

Dewey, J. (1927). The public and its problems. Chicago: Swallow.

Douglas, M. (1983). Risk and culture: an essay on the selection of technical and environmental dangers. Londres: University of California Press.

Dozier, D. Gruning, J. y Gruning L. (1995). The manager's guide to excellence in public relations and communication management. Mahwah, NJ: Lawrence Erlbaum Publishers.

Durandin, G. (1995). La mentira en la propaganda politica y en la publicidad. Barcelona: Paidos.

Fita, J. (1999). Comunicación en programa de crisis. Barcelona: Gestión 2000.

Fraguas, M. (1985). Teoría de la desinformación. Madrid: Alhambra.

Garcia Pelayo, M. (1994). Las transformaciones del Estado de la sociedad civil. Madrid: Alianza Editorial.

Gigliotti, R. y Jason, R. (1991). Emergency planning for maximum protection. Massachussetts: Butter-Heinemann.

Habermas, J (2009). El futuro de la naturaleza humana. Madrid: Paidós.

Hefron, F. (1989). Organization theory and public organizations, the political connection. Englewood Cilffs: Prentice Hall.

La Porte, J. (2012). Introducción a la Comunicación Institucional de las Iglesia. Madrid: Pelícano.

Lazarsfel, P. y Katz, E. (1922). Personal influence: the part played by people in the flow of mass communications. Nueva York: Columbia University.

Lippman, W. (1922). Public opinión. Nueva York: Harcourt Brace Jovanovich.

Mills, C. W. (1956) The power elite. Oxford: Oxford University Press

Lucas Marín, A. (1996). La comunicación en empresas y organizaciones. Barcelona: Bosch.

Mayo, A. (1991). Managing careers. Strategies for organizations. Londres: Institute of personnel management. 
Porto Simoes, R. (1993) Relaciones Públicas: función política. En la empresa y en la institución pública. Barcelona: El Ateneo.

Ramírez, T. (1995). Gabinetes de comunicación. Funciones, disfunciones e incidencias. Barcelona: Bosch.

Sauvy, A. (1970). La opinión pública. Barcelona: oiko-tau.

Schattschneider, E. E. (1960). The semisovereing people: a realist's view of democracy in America. Nueva York: Holt, Rinehart y Winston.

Serrano, E (1994). Legitimación y Racionalización: Weber y Habermas: La Dimensión Normativa de un orden secularizado. Madrid: Anthropos

Sotelo Enriquez, J. (2001). Introducción a la comunicación institucional. Barcelona: Ariel.

Weber, M. (1984). Los tipos de Dominación. México: Centro Latinoamericano de Economía Humana.

Weil, P. (1992). La comunicación global. Comunicación institucional y de gestión. Barcelona: Paidós.

Westphalen, M. (1994). Le communicator. Guide opérationnel pour la communication d'entreprise. París: Dunod.

\section{María de las Mercedes Cancelo Sanmartín}

Doctora en Ciencias de la Comunicación por la Universidad de Málaga, España, Posgrado en Gestión de Políticas Públicas por la Universidad Nacional de la Plata, Argentina. Ha sido profesora visitante en diversas universidades nacionales e internacionales, así como en centros de investigación. Asimismo ha desarrollado extensamente líneas de investigación que versan sobre Comunicación en Fuerzas de Seguridad del Estado, y Nuevas Tecnologías de la Información. Participación en numerosos congresos nacionales e internacionales como ponente y conferencista magistral invitada en Argentina, Estados Unidos, España, México y Portugal. Pertenece a numerosas asociaciones nacionales e internacionales tales como: DIRCOM, ECREA, FISEC, CONAC, AIEC.

\section{Gilda Gadea Aldave}

Máster en Unión Europea por el Instituto Europeo (España), Maestría en Comunicación por la Universidad Autónoma de Tamaulipas (México), Diplomado en Análisis Político (México) y Licenciada en Relaciones Internacionales (México). Sus líneas de investigación son seguridad internacional, grupos paramilitares, migraciones internacionales, propaganda política en regímenes totalitarios y relaciones cívicomilitares. Ha realizado diversas publicaciones y disertaciones a nivel nacional e internacional. Directora y Fundadora de la revista Política y Jurídica, y Presidente de European Center for Social Science Investigation (ECSSI). Investigadora del área de 
Comunicación - Institucional - Redes - Inseguridad - Medios -Ciudadanía

formación académica del Centro de Investigación BCUAT en la Universidad Autónoma de Tamaulipas (México). 